\title{
Key roles for lipid mediators in the adaptive immune response
}

\author{
Parker F. Duffney, ${ }^{1,2}$ Megan L. Falsetta,, ${ }^{1,2}$ Ashley R. Rackow, ${ }^{1,2}$ Thomas H. Thatcher, ${ }^{2,3}$ Richard P. Phipps, $^{1,2,3}$ and Patricia J. Sime ${ }^{1,2,3}$ \\ 'Department of Environmental Medicine, ${ }^{2}$ Lung Biology and Disease Program, and ${ }^{3}$ Division of Pulmonary and Critical Care Medicine, University of Rochester School of Medicine and Dentistry, Rochester, \\ New York, USA.
}

\begin{abstract}
Chronic inflammation is an underlying feature of many diseases, including chronic obstructive pulmonary disease, rheumatoid arthritis, asthma, and multiple sclerosis. There is an increasing appreciation of the dysregulation of adaptive immunity in chronic inflammatory and allergic diseases. The discovery of specialized pro-resolving lipid mediators (SPMs) that actively promote the resolution of inflammation has opened new avenues for the treatment of chronic inflammatory diseases. Much work has been done focusing on the impact of SPMs on innate immune cells. However, much less is known about the influence of SPMs on the development of antigen-specific adaptive immune responses. This Review highlights the important breakthroughs concerning the effects of SPMs on the key cell types involved in the development of adaptive immunity, namely dendritic cells, T cells, and B cells.
\end{abstract}

\section{Introduction}

Inflammation is an inherently beneficial process that recruits immune cells to the site of tissue damage or infection and is sometimes followed by an eventual return to homeostasis. However, many diseases, including chronic obstructive pulmonary disease (1-3), rheumatoid arthritis $(4,5)$, asthma (2), and multiple sclerosis $(6,7)$, are characterized by persistent, nonproductive inflammation that fails to resolve. It is now recognized that a novel class of lipid mediators derived mainly from dietary polyunsaturated fatty acids (PUFAs) contribute to the resolution of inflammation. These mediators, collectively called specialized pro-resolving lipid mediators (SPMs), include families of compounds termed "resolvins," "lipoxins," "maresins," and "protectins" (8). The resolution phase of a normal inflammatory response is characterized by lipid mediator "class switching," in which cells downregulate enzymes responsible for the production of proinflammatory lipids, such as prostaglandins and leukotrienes, while upregulating enzymes responsible for the production of SPMs (9). SPMs are unique in that they exert proresolving and antiinflammatory effects without suppressing the immune response (reviewed in refs. 9 and 10). This is in contrast to classical antiinflammatory therapies, including corticosteroids and nonsteroidal antiinflammatory drugs (NSAIDs). SPMs have exciting therapeutic potential because they exert potent effects in the nanomolar to picomolar range and are made endogenously by many cells. Therefore, they are generally regarded as nontoxic and are likely to be well tolerated when supplied exogenously.

Many SPMs have been found to exert their actions in a receptordependent manner (11). To date, five $G$ protein-coupled receptors have been identified that can bind SPMs: the lipoxin A4 (LXA4) receptor (ALX, also known as formyl peptide receptor 2 [FPR2]), GPR32 (also known as D resolvin receptor 1 [DRV1]), GPR18 (also

Conflict of interest: The authors have declared that no conflict of interest exists. Reference information: / Clin Invest. 2018;128(7):2724-2731.

https://doi.org/10.1172/JCI97951. known as the E resolvin receptor ERV1), ChemR23 (also known as D resolvin receptor 2 [DRV2]), and leukotriene B4 receptor 1 (BLT1). Despite this, the receptors for many SPMs remain to be uncovered. SPM receptors show stereoselective and SPM-specific affinities (reviewed in ref. 11). While knockout and overexpression studies have shown the necessity of receptor-mediated signaling for the pro-resolving effects of SPMs, the downstream signaling events remain poorly understood. SPMs can act as inhibitors of proinflammatory signals, as is the case with resolvin E1 (RvE1), which antagonizes BLT1 activation by leukotriene B4 (12). ALX mediates the proresolving actions of LXA4, resolvin D1 (RvD1), and resolvin D3 (RvD3), yet is also involved in proinflammatory signaling in response to serum amyloid A $(11,13)$. Cooray et al. demonstrated that the formation of ALX homodimers preferentially responds to pro-resolving signals (14). While the pathways activated by SPMs are still poorly characterized, RvE1 enhances the phosphorylation of AKT and phosphorylation of the downstream target rS6 (15). The complexities of SPM signaling remain to be understood and likely involve many receptor-specific mechanisms.

SPMs are effective in resolving acute inflammation in many disease models, including peritonitis (16), asthma (17), periodontitis (18), and colitis (19). Some of the earliest work on SPMs involved the discovery that lipoxins, synthesized from the same arachidonic acid substrate as the (generally) proinflammatory prostaglandins, promote the resolution of inflammation (20). Subsequently, the role of SPMs in the maintenance of homeostasis, the regulation of inflammatory responses, and the regulation of innate and adaptive immune responses has been the focus of considerable study.

Adaptive immune responses are triggered following the activation of innate immune cells in the presence of a danger signal. Innate cells produce inflammatory cytokines, leukotrienes, and prostaglandins that recruit granulocytes and antigen-presenting cells (APCs) to the site of injury. These cells can process and display antigen to activate adaptive immune cells (i.e., T cells and $\mathrm{B}$ cells), stimulating antibody production and subsequent removal of 


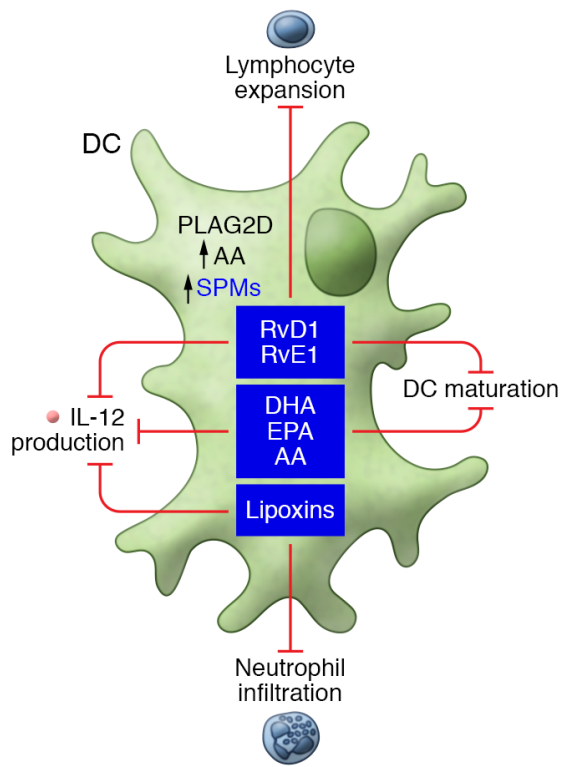

Figure 1. SPMs regulate DC activation markers, including suppression of cytokine production and expression of MHC and costimulatory markers that promote a return to homeostasis following an acute inflammatory response.

the inflammatory stimuli. As the insult is addressed, cells undergo "lipid mediator class switching," a process that is spatially and temporally regulated to suppress further inflammation and stimulate the removal of inflammatory cells and debris. The ideal outcome of these events is a return to tissue homeostasis. The effect of SPMs on innate inflammatory responses and innate immune cell function is well studied and is reviewed elsewhere (9). Here, we will review the effects of SPMs on the cells of the adaptive immune system and consider how these SPMs might be used to promote health and beneficial immune responses that may ultimately help to dampen chronic and pathologic immune responses.

\section{Endogenous sources of SPMs involved in adaptive immunity}

Relatively little is known about the endogenous sources of SPMs in normal immune responses. SPMs are found in human peripheral blood $(21,22)$, bronchoalveolar lavage fluid (23), sputum (24), exhaled breath condensates $(23,25)$, tears $(26)$, spleen $(21)$, lymph nodes $(21)$, brain $(27,28)$, cerebrospinal fluid $(28,29)$, adipose tissue (30), placenta (31), synovial fluid (4), breast milk $(32,33)$, and urine (34). However, within these fluids or tissues, the specific cell types responsible for the production of SPMs are unclear. For example, mouse spleen contains RvD1 and its precursor, 17-hydroxydocosahexaenoic acid (17-HDHA) (35), placing these mediators in close contact with their B cell targets in the maturation of an antibody response; yet the precise cellular source ( $\mathrm{T}$ cells, stromal cells, APCs, etc.) is still unknown. In a self-limited allergic model, mouse lungs contained maresin-1 (MaR1) that reduced expression of IL-5 and IL- 13 by type 2 innate lymphoid cells (ILC2s) and promoted the activity of regulatory $\mathrm{T}$ cells (Tregs), but the specific cellular source of the MaR1 was not identified (36). Synthesis of SPMs by cells of the adaptive immune system is also currently poorly documented. In one interesting example, peripheral blood mononuclear cells
(PBMCs) driven to a Th2 phenotype by concanavalin A and IL-4 produce protectin D1 (PD1), while PBMCs driven to Th1 by IFN- $\gamma$ do not (37). It is understood that B cells, T cells, and dendritic cells (DCs) communicate with each other and with cells of the innate immune system, such as neutrophils, within peripheral tissues, lymph nodes, the spleen, and bone marrow; it is likely that this communication includes the exchange of SPMs. SPM synthesis can be extremely complicated, involving a process termed "transcellular biosynthesis," in which one cell type produces inactive precursors that are handed off to a second cell type to be converted to active mediators $(38,39)$. This process may also play a role in cell-cell communication during inflammation and resolution. The increasing sensitivity of SPM detection methods makes further discoveries in this area inevitable.

\section{SPMs downregulate DC maturation and function}

The development of antigen-specific immune responses starts with the processing and display of immunogenic antigens to adaptive immune cells. DCs serve as professional APCs, surveying the physiologic environment to support the adaptive immune system. To gain functionality, DCs must undergo activation and maturation, characterized by a reduced phagocytic capacity and enhanced antigen processing via the expression of costimulatory molecules (40). Mature DCs carry antigen from the periphery to a draining lymph node, inducing the adaptive immune system. DC maturation is critical for $\mathrm{T}$ cell expansion and differentiation, allowing $\mathrm{T}$ cells to become activated by making contact at the immunological synapse. DCs can also activate naive and memory B cells through their ability to stimulate $\mathrm{CD} 4^{+} \mathrm{T}$ cells. This can further induce $\mathrm{B}$ cell growth and support B cell memory formation in germinal centers (40).

SPMs have demonstrated effects on DC maturation: they affect the ability of DCs to migrate and to produce cytokines, two key features that are required for proper antigen presentation and the priming of $\mathrm{T}$ cell responses (Figure 1). In addition, the application of SPMs targeting DCs may represent a novel mechanism by which cells reduce inflammation and antigen presentation after the injurious stimuli has been removed. This limits lymphocyte priming and promotes the return to homeostasis.

Mononuclear APCs, including DCs, highly express ALX/ FPR2, the receptor for LXA4, RvD1, and RvD3, and agonists for this receptor reduce neutrophil infiltration, decrease the secretion of proinflammatory cytokines, and promote resolution $(41,42)$. Mouse splenic CD11 $\mathrm{c}^{+}$DCs treated with LXA4 have decreased IL-12 production in response to Toxoplasma gondii infection (43). Following LPS stimulation, immature bone marrow-derived dendritic cells (BMDCs) treated with an RvD1 analog expressed reduced levels of MHC class II, CD40, and IL-12, but not CD80/ CD86. This suggests that SPMs, such as RvD1, may modulate DC-T cell interactions through the CD40/154 pathway, but not the CD80/86-CD28 pathway (44). The SPM precursors docosahexaenoic acid (DHA) (45), eicosapentaenoic acid (EPA) (46), and arachidonic acid (46) also inhibit BMDC maturation and IL-12 production in vitro. Importantly, in an allogeneic corneal graft model, an RvD1 analog suppressed allosensitization, reduced the number of IFN- $\gamma$-secreting T cells, and increased graft survival time (44). This indicates that RvD1 is a strong candidate for therapeutic interventions targeted at preventing graft rejection. 
RvE1 has demonstrated similar effects on DC maturation. BMDCs treated with RvE1 remain in an immature state, failing to upregulate costimulatory molecules following TLR stimulation and exhibiting decreased IL-12 secretion. After RvE1 exposure, BMDCs also fail to upregulate CCR7, thus preventing their migration to the draining lymph node (19). As a result, these DCs remain in the region of inflammation, resulting in reduced lymphocyte expansion. RvE1 also inhibits the cutaneous migration of DCs (47), resulting in reduced $\mathrm{T}$ cell responses. In addition, BMDCs generated in the presence of RvE1 inhibit T cell cytokine secretion and increase apoptosis in antigen-bearing T cells (48). From these studies, it is clear that RvE1 modulates the actions of DCs, encouraging resolution by decreasing the frequency of lymphocyte expansion and increasing apoptosis in local antigen-bearing $\mathrm{T}$ cells at the site of inflammation.

Prostaglandins and lipoxins are derived from the precursor arachidonic acid, which is liberated from plasma membranes by phospholipase $\mathrm{A}_{2}\left(\mathrm{PLA}_{2}\right)$ (49). Although several members of the secreted phospholipase $\mathrm{A}_{2}\left(\mathrm{sPA}_{2}\right)$ family have been established as proinflammatory mediators (50), other members of the same family have pro-resolving functions $(51,52)$. For example, group IIA sPLA 2 family members contribute to host defense by degrading bacterial membranes (53), while group V sPLA family members kill fungi by promoting phagocytic uptake by macrophages (52). PLA2G2D is a group IID phospholipase within the PLA family that is preferentially expressed by macrophages and CD $11 \mathrm{c}^{+} \mathrm{DCs}$. Knockout mice lacking PLA2G2D experience delayed resolution of hapten-induced contact dermatitis, and this phenotype can be transferred by adoptive transfer of PLA2G2D-deficent BMDCs to wild-type mice. PLA2G2D-knockout mice also express lower levels of RvD1 and its precursor, 17-HDHA, and PLA2G2D-deficient DCs express higher levels of MHC class II and IL-12 (54). The inference is that PLA2G2D promotes synthesis of pro-resolving mediators, possibly via arachidonic acid, so that PLA2G2D deficiency leads to increased inflammation and delayed resolution. Taken together, these reports show that SPMs are primarily downregulators of DC function, producing reductions in $\mathrm{T}$ cell activation and inflammation. Interestingly, expression of ALX/FPR2 is reduced on mature DCs, suggesting that SPMs play a larger role in regulating DC maturation than in regulating mature DCs (44). While reduction of DC maturation could be interpreted as an immunosuppressive outcome, it is important to remember that the resolution of inflammation is temporally regulated. SPMs are produced to trigger the resolution of existing inflammation, and thus, downregulation of DC maturation represents the termination of the proinflammatory program and a step toward the restoration of homeostasis.

\section{SPMs modulate T cell phenotype and cytokine production}

T cells serve a wide array of roles in both the regulation and resolution of inflammation and adaptive immune responses. While $\mathrm{CD}^{+}$ $\mathrm{T}$ cells are known for their ability to kill virally infected cells, CD4 ${ }^{+}$ $\mathrm{T}$ cells are classically stratified into four classes: type $1 \mathrm{~T}$ helper (Th1), type 2 T helper (Th2), T helper 17 (Th17), and Tregs. CD4 ${ }^{+}$ $\mathrm{T}$ cell subsets are determined by the expression of distinct surface receptors, transcription factors, and distinct cytokine responses, as a result of stimulation (55). T cells perform a wide array of func- tions in the development of long-term immune defense, including the production of inflammatory cytokines, regulation of B cell differentiation, and removal of damaged or infected cells (56). Proper activation and differentiation of $\mathrm{T}$ cell subsets is critical to proper resolution of inflammation. Indeed, many disease states, including asthma (57), rheumatoid arthritis (58), and multiple sclerosis (59), have been linked to dysregulation of proper $\mathrm{T}$ cell differentiation and function.

The ability of bioactive lipids to modulate $\mathrm{T}$ cell function is an active area of research. While work has shown that SPMs have beneficial effects in diseases linked to T cell dysfunction, the direct effects of SPMs on $\mathrm{T}$ cell function are only beginning to be understood. Early studies showed that long-chain omega-3 PUFAs, now known to be precursors for SPMs, can inhibit $\mathrm{T}$ cell proliferation and cytokine production (60). Supplementation of mouse diets with fish oil or DHA increased $\mathrm{CD}^{+} \mathrm{T}$ cell proliferation in response to a Th2 stimulus and decreased Th2 cytokine production in $\mathrm{CD}^{+}$ $\mathrm{T}$ cells (61). T cells also express known SPM receptors, including ALX/FPR2 (62, 63), GPR32 (63), and BLT1 (64), which confirms that they can be targeted by SPMs. SPMs are present in the lymph node (21) and thus are likely to come in contact with T cells. Th2skewed T cells can also produce PD1, thereby highlighting the potential role of SPMs in T cell regulation (37).

One key function of $\mathrm{T}$ cells is the production of helper cytokines that can stimulate further activation of T cells, B cells, and DCs, and there is evidence that SPMs act to suppress this activation. PD1 reduced the production of IFN- $\gamma$ and TNF- $\alpha$ by Th1 and Th17 CD4 ${ }^{+} \mathrm{T}$ cells (37). Recent studies showed that RvD1, RvD2, and MaR1 could similarly block the production of IFN- $\gamma$ and TNF- $\alpha$ by T cells (63). The effect was similar on both $\mathrm{CD} 4^{+}$and $\mathrm{CD} 8^{+}$cells, suggesting that SPMs can regulate multiple subsets of T cells. The suppression of IFN- $\gamma$ production in T cells is shared among many SPMs; RvD3, LXA4, lipoxin B4 (LXB4), and even SPM derivatives were effective at blocking TNF- $\alpha$ production in $\mathrm{CD} 4^{+} \mathrm{T}$ cells $(62$, 63). Thus, it is clear that many SPMs can attenuate inflammatory cytokine production by $\mathrm{T}$ cells.

The differentiation of $\mathrm{CD} 4^{+} \mathrm{T}$ cells into various Th subtypes is critical to stimulate inflammatory responses. Chiurchiu et al. showed that RvD1, RvD2, and MaR1 suppressed the differentiation of naive $\mathrm{T}$ cells into Th1 and Th17 subtypes while increasing the production of Tregs (63). Tregs are known for their ability to produce pro-resolution factors to control inflammation, such as TGF- $\beta$ (55). Indeed, Tregs that were augmented by MaR1 treatment were able to suppress inflammatory cytokine production in ILC2s (36). This SPM skewing of cell lineage to favor Treg production has also been observed in animal models. RvD1 treatment increased Treg numbers, which corresponded to a decreased percentage of Th1 and Th17 cells in a mouse model of multiple sclerosis (6). Mice deficient in Elovl2 (encoding the enzyme elongation of very-long-chain fatty acids protein 2, which can generate SPM precursor lipids de novo) have an increased percentage of Th1 and Th17 T cells and a reduced percentage of Tregs, a phenotype that can be reversed by dietary supplementation with DHA or intraperitoneal RvD1 injection (63). Interestingly, most SPMs show little effect on Th2 T cells, except for RvE1, which can reduce cytokine production by Th2 cells $(65,66)$. These studies demonstrate that SPMs promote a Treg phenotype while attenuating the differen- 


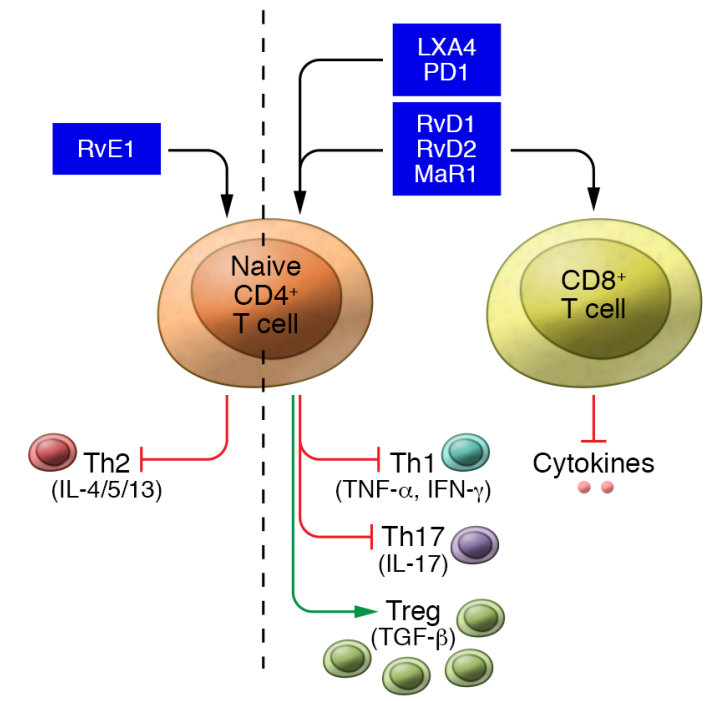

Figure 2. SPMs act on T cells to reduce activation and production of proinflammatory cytokines and upregulate a regulatory $T$ cell phenotype.

tiation and function of inflammatory $\mathrm{T}$ cell subsets. Continued work is needed, as there may be differences in the effects of specific SPMs on the various $\mathrm{T}$ cell subsets.

The resolution of inflammation also requires the clearance of apoptotic cells and a reduction in the amount of proinflammatory signals. $\mathrm{T}$ cells bind and sequester chemokines through the chemokine receptor CCR5, the abundance of which increases during apoptosis (67). Mice lacking this receptor have increased chemokine levels in inflammatory exudates (67). Interestingly, treatment

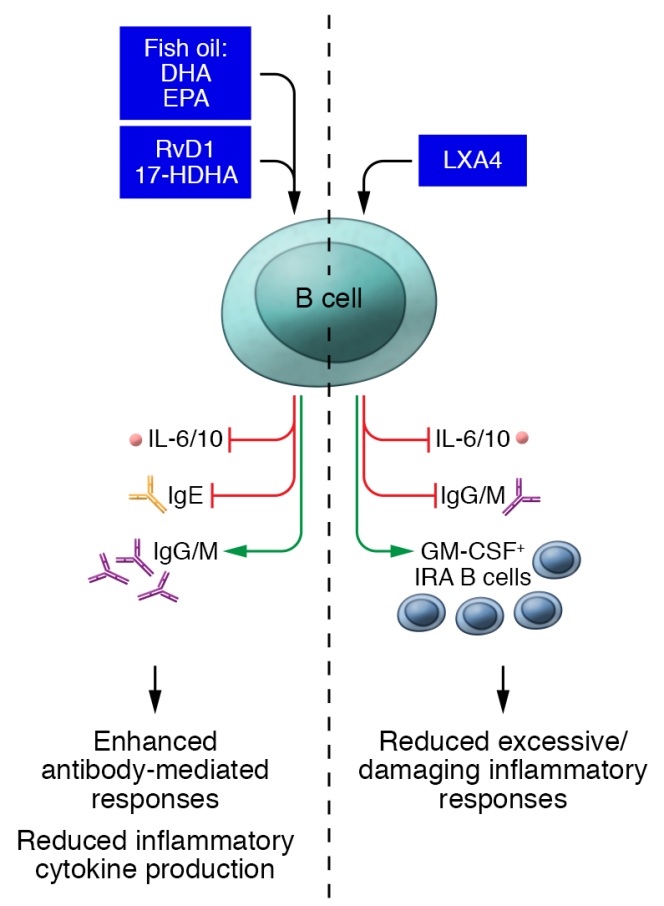

Figure 3. SPMs can either up- or downregulate antibody production depending on the B cell subset and specific SPM involved. SPMs also frequently decrease cytokine production (as depicted), but it is important to note that some SPMs can also stimulate cytokine release in other cell types. with structural analogs of RvE1, LXA4, and PD1 increased the expression of CCR5 on late apoptotic $\mathrm{T}$ cells, suggesting that SPMmediated upregulation of CCR 5 could be a mechanism by which enhanced clearance of inflammatory cytokines occurs during the resolution of inflammation. PD1 also increases T cell apoptosis and could further contribute to enhanced CCR5-induced sequestering of inflammatory mediators (37).

Comprehensive comparison of the effects of different classes of SPMs on T cell functions is still needed. However, it is clear that SPMs can regulate $\mathrm{T}$ cell responses by directing maturation of naive $\mathrm{CD}^{+} \mathrm{T}$ cells to a proregulatory phenotype and increasing the scavenging of inflammatory mediators, all while suppressing inflammatory mediator production in already mature Th1 and Th17 $\mathrm{T}$ cell subsets (Figure 2).

\section{SPMs and PUFAs affect $B$ cell function}

Although the canonical role of B cells is antibody production, $\mathrm{B}$ cells play several important roles in both innate and adaptive immunity. B cells can function as APCs and directly respond to innate and adaptive stimuli via the production of cytokines (68). There are ample scientific data to show that these critical B cell functions are affected by SPMs and their precursor PUFAs (Figure 3). Supplementation with SPMs or PUFAs has been shown to augment $\mathrm{B}$ cell functions by (a) increasing antibody production (35, 69-72), (b) altering B cell cytokine production (69, 72-75), (c) skewing B cell differentiation/lineage (35, 70, 76-78), and (d) limiting spontaneous IgE class switching implicated in allergic asthma $(76,77)$. In addition, SPMs may represent promising and effective adjuvants for vaccination because of their potent effects on B cells (70).

A first clue that SPMs might affect $B$ cell functions was the discovery that B cells express the ALX/FPR2 receptor that recognizes the D-series resolvins, RvD1 and RvD3 (79). 17-HDHA, RvD1, and PD1 are naturally produced within the spleen, a site where B cells commonly reside (35). PUFAs present in omega-3 fatty acid-rich fish oil (precursors for SPM production) were shown to affect B cell functions in mice by increasing antibody production and B cell activation $(71,72,74)$. Similarly, mice fed a diet rich in DHA and EPA had a higher number of IgM-expressing splenic B cells following antigen stimulation compared with mice not receiving a PUFAenriched diet (71). In a mouse model of diet-induced obesity, mice fed a high-fat (Western) diet containing primarily omega-6 fatty acids exhibited diminished antibody titers and increased mortality to influenza challenge relative to a normal diet. These effects could be rescued with dietary DHA (69). Dietary supplementation with PUFAs or PUFA-enriched fish oil also resulted in changes in B cell lipid composition $(71,72,74)$, indicating that these PUFAs were incorporated into the cellular membrane and could also act as a reservoir for the generation of SPMs. More recently, it was determined that dietary supplementation with DHA enhances splenic levels of the SPM precursors 14-HDHA and 17-HDHA (69), and that increased levels of SPMs are seen in the circulation of those taking essential fatty acid supplements $(21,22)$. These data indicate that dietary DHA can be processed to enrich SPM levels. Indeed, DHA supplementation in mice did not directly target $B$ cells but increased splenic levels of 17-HDHA, 14-HDHA, protectin DX (PDX), and RvD2, highlighting the importance of SPMs in reg- 
ulating B cell function. These findings suggest that the beneficial effects of PUFA supplementation are mediated by the enhanced production of SPMs (69).

Several studies have investigated the direct effects of exogenous SPM treatment on B cell function. Addition of exogenous RvD1 and 17-HDHA to human B cells in vitro increased levels of IgM and IgG, while reducing production of the proinflammatory cytokine IL-6 (35). RvD1 and 17-HDHA enhanced B cell differentiation to antibody-secreting cells (ASCs) without increasing the amount of antibody synthesized per ASC or altering B cell proliferation. The end result was a net increase in the amount of IgG and IgM produced (35). Similarly, mice treated with 17-HDHA had increased levels of serum IgG and IgM after challenge with ovalbumin (OVA) (70). The implications of these findings are that enhanced antibody production should lead to more rapid/efficient antigen clearance, thus helping to clear pathogens and resolve inflammation. The use of SPMs to stimulate ASC differentiation can also be exploited to stimulate the generation of antigen-specific antibody responses to vaccination. In a mouse model of influenza vaccination, coinjection of the influenza viral hemagglutinin (HA) protein with 17-HDHA resulted in higher protective antibody titers compared with injection of HA alone. Increased antibody production corresponded to reduced mortality in response to subsequent influenza infection (70). Taken together, these data suggest the exciting concept that 17-HDHA might be a highly useful, nonimmunogenic vaccine adjuvant.

Interestingly, distinct B cell subsets are affected uniquely by different SPMs. LXA4, an SPM generated from arachidonic acid, enhances early antibody responses to novel antigens by increasing the number of $\mathrm{GM}^{-\mathrm{CSF}^{+}}$innate response activator (IRA) B cells and accelerating the migration of $\mathrm{C}^{+} 9^{+} \mathrm{B}$ cells to the spleen (73). In addition, LXA4 inhibits regulatory B cell (Breg) production of the immunosuppressive cytokine IL-10 (80) and decreases the maturation of resting memory B cells to ASCs (78). These findings suggest that LXA4 promotes effective immune responses to new antigens by directing activation of IRA B cells, suppressing Bregs, and inhibiting the maturation of memory B cells, which otherwise would tend to target prior pathogens that may be currently irrelevant. Thus, SPMs may be able to contextually control the generation of new ASCs and the activation of existing memory B cells.

The differential effect of LXA4 and LXB4 on memory and nonmemory B cells could be due to different levels of receptor expression or to coexpression of alternate SPM receptors. Like RvD1, LXA4 binds to the ALX/FPR2 receptor to initiate downstream signaling. ALX/FPR2 is expressed on CD19+ B cells, and its expression can be induced upon activation (78), potentially leading to enhanced signaling in activated B cells. Additionally, some B cell populations also express the SPM receptors ChemR23 and BLT1 (69). The receptor for LXB4 is currently unknown. Further identification of SPM receptors and expression in various B cell subsets will enhance our understanding of the role of SPMs in regulating antibody responses.

Antibody class switching is an important regulatory event that controls effector cell function. For example, IgE is an essential element in allergic diseases. Patients with asthma have elevated circulating levels of B cell-derived IgE (76). 17-HDHA and RvD1, which enhance human B cell IgG production, also suppress the production of IgE. 17-HDHA and RvD1 inhibit B cell class switching to IgE by stabilizing the regulator protein BCL-6, which then prevents the transcription factor STAT6 from binding to the $\varepsilon$-germline transcript ( $\varepsilon$ GLT) promoter (76). This ultimately results in a substantial decrease in $\varepsilon$ GLT expression, which is essential for IgE class switching $(76,77)$. This process occurs both in B cells stimulated with IL-4 and in unstimulated spontaneously IgE-producing B cells collected from donors with allergic asthma. This suggests that, unlike widely used corticosteroids, SPMs (e.g., 17-HDHA and RvD1) might be useful in treating asthma and other allergic diseases without suppressing IgG antibody responses to such challenges as seasonal flu vaccine or new infections.

\section{Summary and future directions}

The discovery that SPMs can stimulate the resolution of inflammation has made them very promising therapeutic candidates. Indeed, long-acting SPM derivatives have been well tolerated in clinical trials (discussed below). Resolution requires the cooperation of multiple cell types to link acute innate inflammation to the development of long-term, antigen-specific responses. Despite the widespread benefit seen in animal models of acute inflammation, the mechanisms by which SPMs exert their pro-resolving actions remain an active area of investigation. Given their importance in autoimmune and inflammatory diseases, characterizing the effects of SPMs on adaptive immune responses will prove beneficial in targeting adaptive immune defects.

Overall, SPMs show great promise in regulating adaptive immune responses. Receptors for SPMs are found on DCs, T cells, and B cells, and SPMs are produced naturally at sites where they readily interface with adaptive immune cells. Furthermore, exogenous SPM treatment has shown significant beneficial effects on cell functions, ultimately augmenting pathogen clearance, the resolution of inflammation, and the development of immune memory. Future studies should consider comparisons of different classes of SPMs' immune cell functions, as individual SPMs may stimulate unique pro-resolution pathways. Complicating this is the emerging evidence that SPMs can differentially affect distinct subsets of immune cells (e.g., Th1 T cells versus Tregs). Future studies need to address the role of SPMs in regulating heterogeneous populations of immune cells generated during inflammation. The role of SPMs in regulating immune cell maturation as well as the effects on already differentiated cell subsets will be critical to understanding the pro-resolution program. Advancements in techniques to detect SPMs and the identification of new SPM receptors will help clarify the role of specific SPMs in cell type-specific actions.

Temporal regulation of SPM production is key to timely and efficient resolution of inflammation, yet the majority of studies focus on single time points. While indices have been developed to characterize the resolution of inflammation (16), these depend on neutrophil recruitment and clearance from the inflamed site. The generation of indices to account for restoration of adaptive immune responses is still needed. More time-course analyses of SPM generation and subsequent pro-resolving effects will further the understanding of resolution programs. Indeed, understanding the cellular mechanisms involved in SPM-controlled resolution will serve to advance the field of SPM research in the clinical arena.

While exogenous SPM treatments are well tolerated in animal models, potentially unwanted side effects need to be considered. 
Metabolism of DHA can contribute to endothelial barrier function in diabetic retinopathy (81). Similarly, EPA and DHA epoxide metabolites have been shown to promote degranulation of mast cells, which would be detrimental in treating allergic diseases (82). It is thus important to consider the potential effects of SPM metabolites on host responses, especially when translating the findings of animal models to a clinical setting, as well as during the development of novel, metabolically resistant SPM derivatives.

Even with this caveat, development of novel therapeutics that promote resolution is already under way. An RvE1 analog is currently in phase II clinical trial for dry eye (ClinicalTrials.gov identifier NCT00799552). Similarly, the lipoxin analog BLXA4-Me is in a clinical trial to treat gingivitis (ClinicalTrials.gov identifier NCT02342691). While no clinical trials have investigated the actions of SPMs on adaptive immune responses, identification of novel SPM derivatives that target adaptive immune cells holds increasing potential to treat many inflammatory and allergic conditions. Similarly, there is also promise in harnessing the potential of SPMs as adjuvants to increase antibody production to increase the efficacy of vaccination. This is of particular interest in the context of susceptible populations such as infants, the elderly, and immunosuppressed individuals who have reduced capacity to mount effective adaptive immune responses. There are currently few vaccine adjuvants in use, and most vaccines do not contain adjuvants. The development of new adjuvants that can increase antigen-specific antibody production remains direly needed. Activation of pro-resolving adaptive immune responses provides new therapeutic targets to combat inflammatory and allergic diseases. Future work to understand the mechanism of SPM-mediated control of adaptive immune responses will further the advancement of novel therapeutics in the clinical arena.

\section{Acknowledgments}

This work was supported in part by NIH grants T90DE021985, T32HL066988, T32ES007026, R01HL120908, R01HD069313, R21AI103690, and P30ES001247; the Mary Parkes Asthma Center; the C. Jane Davis and C. Robert Davis Professorship (to P.J. Sime); and the Wright Family Professorship (to R.P. Phipps).

Address correspondence to: Patricia J. Sime, University of Rochester, Division of Pulmonary and Critical Care Medicine, Department of Medicine, 601 Elmwood Avenue, Box 692, Rochester, New York 14642, USA. Phone: 585.275.6526; Email: Patricia_ Sime@urmc.rochester.edu.
1. Hsiao HM, Thatcher TH, Colas RA, Serhan CN, Phipps RP, Sime PJ. Resolvin D1 reduces emphysema and chronic inflammation. Am J Pathol. 2015;185(12):3189-3201.

2. Papaiwannou A, et al. Asthma-chronic obstructive pulmonary disease overlap syndrome (ACOS): current literature review. J Thorac Dis. 2014;6(suppl 1):S146-S151.

3. Barbu C, Iordache M, Man MG. Inflammation in COPD: pathogenesis, local and systemic effects. Rom J Morphol Embryol. 2011;52(1):21-27.

4. Giera M, et al. Lipid and lipid mediator profiling of human synovial fluid in rheumatoid arthritis patients by means of LC-MS/MS. Biochim Biophys Acta. 2012;1821(11):1415-1424.

5. McInnes IB, Schett G. The pathogenesis of rheumatoid arthritis. N Engl J Med. 2011;365(23):2205-2219.

6. Poisson LM, et al. Untargeted plasma metabolomics identifies endogenous metabolite with drug-like properties in chronic animal model of multiple sclerosis. J Biol Chem. 2015;290(52):30697-30712.

7. Lassmann H, van Horssen J, Mahad D. Progressive multiple sclerosis: pathology and pathogenesis. Nat Rev Neurol. 2012;8(11):647-656.

8. Serhan CN, Chiang N, Van Dyke TE. Resolving inflammation: dual anti-inflammatory and proresolution lipid mediators. Nat Rev Immunol. 2008;8(5):349-361.

9. Basil MC, Levy BD. Specialized pro-resolving mediators: endogenous regulators of infection and inflammation. Nat Rev Immunol. 2016;16(1):51-67.

10. Serhan CN, Chiang N, Dalli J. The resolution code of acute inflammation: Novel pro-resolving lipid mediators in resolution. Semin Immunol. 2015;27(3):200-215.

11. Chiang N, Serhan CN. Structural elucidation and physiologic functions of specialized pro-resolving mediators and their receptors. Mol Aspects Med. 2017;58:114-129.

12. Arita M, Ohira T, Sun YP, Elangovan S, Chiang $\mathrm{N}$, Serhan CN. Resolvin E1 selectively interacts with leukotriene B4 receptor BLT1 and ChemR23 to regulate inflammation. JImmunol. 2007;178(6):3912-3917.

13. He R, Sang H, Ye RD. Serum amyloid A induces IL-8 secretion through a G proteincoupled receptor, FPRL1/LXA4R. Blood. 2003;101(4):1572-1581.

14. Cooray SN, et al. Ligand-specific conformational change of the G-protein-coupled receptor ALX/FPR2 determines proresolving functional responses. Proc Natl Acad Sci US A. 2013;110(45):18232-18237.

15. Ohira T, Arita M, Omori K, Recchiuti A, Van Dyke TE, Serhan CN. Resolvin E1 receptor activation signals phosphorylation and phagocytosis. J Biol Chem. 2010;285(5):3451-3461.

16. Bannenberg GL, et al. Molecular circuits of resolution: formation and actions of resolvins and protectins. J Immunol. 2005;174(7):4345-4355.

17. Haworth O, Cernadas M, Yang R, Serhan CN, Levy BD. Resolvin E1 regulates interleukin 23 , interferon- $\gamma$ and lipoxin A4 to promote the resolution of allergic airway inflammation. Nat Immunol. 2008;9(8):873-879.

18. Hasturk H, et al. RvE1 protects from local inflammation and osteoclast-mediated bone destruction in periodontitis. FASEB J. 2006;20(2):401-403.

19. Arita M, et al. Resolvin E1, an endogenous lipid mediator derived from omega-3 eicosapentaenoic acid, protects against 2,4,6-trinitrobenzene sulfonic acid-induced colitis. Proc Natl Acad Sci US A. 2005;102(21):7671-7676.

20. Chiang N, Takano T, Clish CB, Petasis NA, Tai $\mathrm{HH}$, Serhan CN. Aspirin-triggered 15-epi-lipoxin A4 (ATL) generation by human leukocytes and murine peritonitis exudates: development of a specific 15-epi-LXA4 ELISA. J Pharmacol Exp Ther. 1998;287(2):779-790.

21. Colas RA, Shinohara M, Dalli J, Chiang N, Serhan CN. Identification and signature profiles for pro-resolving and inflammatory lipid mediators in human tissue. Am J Physiol Cell Physiol. 2014;307(1):C39-C54.

22. Mas E, Croft KD, Zahra P, Barden A, Mori TA. Resolvins D1, D2, and other mediators of selflimited resolution of inflammation in human blood following n-3 fatty acid supplementation. Clin Chem. 2012;58(10):1476-1484.

23. Croasdell A, et al. Resolvins attenuate inflammation and promote resolution in cigarette smokeexposed human macrophages. Am J Physiol Lung Cell Mol Physiol. 2015;309(8):L888-L901.

24. Yang J, Eiserich JP, Cross CE, Morrissey BM, Hammock BD. Metabolomic profiling of regulatory lipid mediators in sputum from adult cystic fibrosis patients. Free Radic Biol Med. 2012;53(1):160-171.

25. Levy BD, et al. Protectin D1 is generated in asthma and dampens airway inflammation and hyperresponsiveness. Jimmunol. 2007;178(1):496-502.

26. English JT, Norris PC, Hodges RR, Dartt DA, Serhan CN. Identification and profiling of specialized pro-resolving mediators in human tears by lipid mediator metabolomics. Prostaglandins Leukot Essent Fatty Acids. 2017;117:17-27.

27. Lukiw WJ, et al. A role for docosahexaenoic acid-derived neuroprotectin D1 in neural cell survival and Alzheimer disease. J Clin Invest. 2005;115(10):2774-2783.

28. Wang X, et al. Resolution of inflammation is altered in Alzheimer's disease. Alzheimers Dement. 2015;11(1):40-50.e1.

29. Prüss H, et al. Proresolution lipid mediators in multiple sclerosis - differential, disease severitydependent synthesis - a clinical pilot trial. PLOS 
One. 2013;8(2):e55859

30. Claria J, Nguyen BT, Madenci AL, Ozaki CK, Serhan CN. Diversity of lipid mediators in human adipose tissue depots. Am J Physiol Cell Physiol. 2013;304(12):C1141-C1149.

31. Keelan JA, et al. Effects of maternal n-3 fatty acid supplementation on placental cytokines, proresolving lipid mediators and their precursors. Reproduction. 2015;149(2):171-178.

32. Weiss GA, Troxler H, Klinke G, Rogler D, Braegger C, Hersberger M. High levels of anti-inflammatory and pro-resolving lipid mediators lipoxins and resolvins and declining docosahexaenoic acid levels in human milk during the first month of lactation. Lipids Health Dis. 2013;12:89.

33. Arnardottir H, Orr SK, Dalli J, Serhan CN. Human milk proresolving mediators stimulate resolution of acute inflammation. Mucosal Immunol. 2016;9(3):757-766.

34. Sasaki A, et al. Determination of omega- 6 and omega-3 PUFA metabolites in human urine samples using UPLC/MS/MS. Anal Bioanal Chem. 2015;407(6):1625-1639.

35. Ramon S, Gao F, Serhan CN, Phipps RP. Specialized proresolving mediators enhance human $\mathrm{B}$ cell differentiation to antibody-secreting cells. J Immunol. 2012;189(2):1036-1042.

36. Krishnamoorthy N, et al. Cutting edge: maresin-1 engages regulatory $\mathrm{T}$ cells to limit type 2 innate lymphoid cell activation and promote resolution of lung inflammation. J Immunol. 2015;194(3):863-867.

37. Ariel A, et al. The docosatriene protectin $\mathrm{D} 1$ is produced by $\mathrm{TH} 2$ skewing and promotes human $\mathrm{T}$ cell apoptosis via lipid raft clustering. J Biol Chem. 2005;280(52):43079-43086.

38. Capra V, Rovati GE, Mangano P, Buccellati C, Murphy RC, Sala A. Transcellular biosynthesis of eicosanoid lipid mediators. Biochim Biophys Acta. 2015;1851(4):377-382.

39. Serhan CN, Clish CB, Brannon J, Colgan SP, Chiang N, Gronert K. Novel functional sets of lipid-derived mediators with antiinflammatory actions generated from omega- 3 fatty acids via cyclooxygenase 2-nonsteroidal antiinflammatory drugs and transcellular processing. J Exp Med. 2000;192(8):1197-1204.

40. Sabado RL, Balan S, Bhardwaj N. Dendritic cellbased immunotherapy. Cell Res. 2017;27(1):74-95.

41. Chen K, et al. A critical role for the g proteincoupled receptor $\mathrm{mFPR} 2$ in airway inflammation and immune responses. Jimmunol. 2010;184(7):3331-3335.

42. Kojima F, Kapoor M, Kawai S, Crofford LJ. New insights into eicosanoid biosynthetic pathways: implications for arthritis. Expert Rev Clin Immunol. 2006;2(2):277-291.

43. Aliberti J, Hieny S, Reis e Sousa C, Serhan CN, Sher A. Lipoxin-mediated inhibition of IL-12 production by DCs: a mechanism for regulation of microbial immunity. Nat Immunol. 2002;3(1):76-82.

44. Hua J, et al. The resolvin D1 analogue controls maturation of dendritic cells and suppresses alloimmunity in corneal transplantation. Invest Ophthalmol Vis Sci. 2014;55(9):5944-5951.

45. Kong W, Yen JH, Vassiliou E, Adhikary S, Toscano MG, Ganea D. Docosahexaenoic acid prevents dendritic cell maturation and in vitro and in vivo expression of the IL-12 cytokine family. Lipids Health Dis. 2010;9:12.

46. Zeyda M, et al. Polyunsaturated fatty acids block dendritic cell activation and function independently of NF- $\mathrm{KB}$ activation. J Biol Chem. 2005;280(14):14293-14301.

47. Sawada Y, et al. Resolvin E1 inhibits dendritic cell migration in the skin and attenuates contact hypersensitivity responses. J Exp Med. 2015;212(11):1921-1930.

48. Vassiliou EK, Kesler OM, Tadros JH, Ganea D. Bone marrow-derived dendritic cells generated in the presence of resolvin E1 induce apoptosis of activated $\mathrm{CD} 4^{+} \mathrm{T}$ cells. JImmunol. 2008;181(7):4534-4544.

49. Serhan CN, Haeggström JZ, Leslie CC. Lipid mediator networks in cell signaling: update and impact of cytokines. FASEB J.1996;10(10):1147-1158.

50. Murakami M, Taketomi Y, Miki Y, Sato H, Hirabayashi T, Yamamoto K. Recent progress in phospholipase A(2) research: from cells to animals to humans. Prog Lipid Res. 2011;50(2):152-192.

51. Weinrauch Y, Elsbach P, Madsen LM, Foreman A, Weiss J. The potent anti-Staphylococcus aureus activity of a sterile rabbit inflammatory fluid is due to a $14-\mathrm{kD}$ phospholipase A2. JClin Invest. 1996;97(1):250-257.

52. Balestrieri B, Maekawa A, Xing W, Gelb MH, Katz HR, Arm JP. Group V secretory phospholipase A2 modulates phagosome maturation and regulates the innate immune response against Candida albicans. JImmunol. 2009;182(8):4891-4898.

53. Wright GC, Weiss J, Kim KS, Verheij H, Elsbach P. Bacterial phospholipid hydrolysis enhances the destruction of Escherichia coli ingested by rabbit neutrophils. Role of cellular and extracellular phospholipases. JClin Invest. 1990;85(6):1925-1935.

54. Miki Y, et al. Lymphoid tissue phospholipase A2 group IID resolves contact hypersensitivity by driving antiinflammatory lipid mediators. J Exp Med. 2013;210(6):1217-1234.

55. Raphael I, Nalawade S, Eagar TN, Forsthuber TG. $\mathrm{T}$ cell subsets and their signature cytokines in autoimmune and inflammatory diseases. Cytokine. 2015;74(1):5-17.

56. Korn T, Bettelli E, Oukka M, Kuchroo VK. IL-17 and Th17 cells. Annu Rev Immunol. 2009;27:485-517.

57. Kuperman DA, et al. Direct effects of interleukin13 on epithelial cells cause airway hyperreactivity and mucus overproduction in asthma. Nat Med. 2002;8(8):885-889.

58. Raphael I, Forsthuber TG. Stability of T-cell lineages in autoimmune diseases. Expert Rev Clin Immunol. 2012;8(4):299-301.

59. Olsson T. Cytokines in neuroinflammatory disease: role of myelin autoreactive $\mathrm{T}$ cell production of interferon- $\gamma$. J Neuroimmunol.1992;40 (2-3):211-218.

60. Costabile $\mathrm{M}$, et al. A novel long chain polyunsaturated fatty acid, beta-Oxa 21:3n-3, inhibits T lymphocyte proliferation, cytokine production, delayed-type hypersensitivity, and carrageenan-induced paw reaction and selectively targets intracellular signals. JImmunol. 2001;167(7):3980-3987.

61. Arrington JL, Chapkin RS, Switzer KC, Morris JS, McMurray DN. Dietary n-3 polyunsaturated fatty acids modulate purified murine T-cell subset acti- vation. Clin Exp Immunol. 2001;125(3):499-507.

62. Ariel A, Chiang N, Arita M, Petasis NA, Serhan

$\mathrm{CN}$. Aspirin-triggered lipoxin A4 and B4 analogs block extracellular signal-regulated kinase-

dependent TNF- $\alpha$ secretion from human T cells. J Immunol. 2003;170(12):6266-6272.

63. Chiurchiu V, et al. Proresolving lipid mediators resolvin D1, resolvin D2, and maresin 1 are critical in modulating $\mathrm{T}$ cell responses. Sci Transl Med. 2016;8(353):353ra111.

64. Tager AM, et al. Leukotriene B4 receptor BLT1 mediates early effector T cell recruitment. Nat Immunol. 2003;4(10):982-990.

65. Aoki H, et al. Protective effect of resolvin E1 on the development of asthmatic airway inflammation. Biochem Biophys Res Commun. 2010;400(1):128-133.

66. Aoki H, et al. Resolvin E1 dampens airway inflammation and hyperresponsiveness in a murine model of asthma. Biochem Biophys Res Commun. 2008;367(2):509-515.

67. Ariel A, et al. Apoptotic neutrophils and T cells sequester chemokines during immune response resolution through modulation of CCR5 expression. Nat Immunol. 2006;7(11):1209-1216.

68. Shaikh SR, Haas KM, Beck MA, Teague H. The effects of diet-induced obesity on B cell function. Clin Exp Immunol. 2015;179(1):90-99.

69. Kosaraju R, et al. B cell activity is impaired in human and mouse obesity and is responsive to an essential fatty acid upon murine influenza infection. JImmunol. 2017;198(12):4738-4752.

70. Ramon S, et al. The specialized proresolving mediator 17-HDHA enhances the antibodymediated immune response against influenza virus: a new class of adjuvant? J Immunol. 2014;193(12):6031-6040.

71. Teague H, Fhaner CJ, Harris M, Duriancik DM, Reid GE, Shaikh SR. n-3 PUFAs enhance the frequency of murine $\mathrm{B}$-cell subsets and restore the impairment of antibody production to a T-independent antigen in obesity. J Lipid Res. 2013;54(11):3130-3138.

72. Teague H, Harris M, Fenton J, Lallemand P, Shewchuk BM, Shaikh SR. Eicosapentaenoic and docosahexaenoic acid ethyl esters differentially enhance B-cell activity in murine obesity. J Lipid Res. 2014;55(7):1420-1433.

73. Cheng Q, et al. Lipoxin A4 protects against lipopolysaccharide-induced sepsis by promoting innate response activator B cells generation. Int Immunopharmacol. 2016;39:229-235.

74. Gurzell EA, Teague H, Harris M, Clinthorne J, Shaikh SR, Fenton JI. DHA-enriched fish oil targets B cell lipid microdomains and enhances ex vivo and in vivo B cell function. J Leukoc Biol. 2013;93(4):463-470.

75. Teague H, Harris M, Whelan J, Comstock SS, Fenton JI, Shaikh SR. Short-term consumption of n-3 PUFAs increases murine IL-5 levels, but IL-5 is not the mechanistic link between n-3 fatty acids and changes in B-cell populations. J Nutr Biochem. 2016;28:30-36.

76. Kim N, Ramon S, Thatcher TH, Woeller CF, Sime PJ, Phipps RP. Specialized proresolving mediators (SPMs) inhibit human B-cell IgE production. Eur J Immunol. 2016;46(1):81-91.

77. Kim N, Thatcher TH, Sime PJ, Phipps RP. Corti- 
costeroids inhibit anti-IgE activities of specialized proresolving mediators on $\mathrm{B}$ cells from asthma patients. JCI Insight. 2017;2(3):e88588.

78. Ramon S, Bancos S, Serhan CN, Phipps RP. Lipoxin A(4) modulates adaptive immunity by decreasing memory B-cell responses via an ALX/ FPR2-dependent mechanism. Eur JImmunol. 2014;44(2):357-369.
79. Spurr L, Nadkarni S, Pederzoli-Ribeil M, Goulding NJ, Perretti M, D’Acquisto F. Comparative analysis of Annexin A1-formyl peptide receptor 2/ALX expression in human leukocyte subsets. Int Immunopharmacol. 2011;11(1):55-66.

80. Wang Z, et al. Lipid mediator lipoxin A4 inhibits tumor growth by targeting IL-10producing regulatory B (Breg) cells. Cancer Lett.
2015;364(2):118-124.

81. $\mathrm{Hu}$ J, et al. Inhibition of soluble epoxide hydrolase prevents diabetic retinopathy. Nature. 2017;552(7684):248-252.

82. Shimanaka Y, et al. Omega-3 fatty acid epoxides are autocrine mediators that control the magnitude of IgE-mediated mast cell activation. Nat Med. 2017;23(11):1287-1297. 